\title{
Use of C1q or C3d Assays in Risk Assessment Before HLAi Renal Transplantation.
}

\section{O Shaw ${ }^{1}$, L Howe 1 , C Martin¹, K Reddi ${ }^{1}$ N Kessaris², L Silas², I Generaldo², D Game²,} A Dorling², R Vaughan ${ }^{1}, \mathrm{~N}_{\text {Mamode}}$.

\section{Aim:}

To investigate the association of donor specific antibody (DSA) able to bind C1q, or fix C3d, on the relevant single antigen beads pre transplant with both post transplant graft survival and incidence of antibody mediated rejection (AMR) following an HLA antibody incompatible (HLAi) renal transplant.

\section{Methods:}

- 42 recipients of an HLAi renal transplant following at least one antibody removal treatment using DFPP to convert a positive flow crossmatch to negative at a single centre between 2005 and 2014 were identified.

- End points of graft failure (death censored) and incidence of biopsy proven AMR were selected.

- The immediate pre treatment sample was tested for DSA using the OneLambda single antigen pan IgG and C1q methods, and the Immucor single antigen pan IgG and C3d methods as per manufacturers instructions.

\section{Results:}

- Patient demographics and overall antibody results are recorded in table 1.

- In summary

- 19/42 did not show complement activity on either kit.

- $22 / 42$ had at least one DSA capable of binding $\mathrm{C} 1 \mathrm{q}$.

- $18 / 42$ had at least one DSA capable of fixing C3d.

- 5/22 C1q antibodies did not show concomitant C3d fixing ability.

- 1/18 C3d fixing antibodies did not show C1q binding.

- 1 C3d positive/C1q negative pre transplant became C1q positive post transplant - graft failed due to AMR

- 5 C1q positive/C3d negative pre transplant - 1 became C3d post transplant failed due to AMR

- 4 became C1q negative post transplant -1 death with functioning graft.

\begin{tabular}{|c|}
\hline Table 1 \\
\hline $\begin{array}{c}\text { Number of Patients } \\
\text { (Total } n=42)\end{array}$ \\
\hline Gender M:F \\
\hline $\begin{array}{c}\text { Number of ABDR } \\
\text { mismatches }\end{array}$ \\
\hline Number of Ab \\
Specificities \\
\hline Overall Graft Loss \\
\hline Lost due to Rxn \\
\hline Time to Loss \\
\hline AMR in 1 st 6 \\
months \\
\hline Ab Class \\
Cl/Cll/Both \\
\hline Total lgG MFI \\
\hline Total C' fixing MFI \\
\hline
\end{tabular}

Complement Negative

19

\section{$6: 13$}

$3.2(1-5)$

$2.6(1-6)$

C1q Positive

22

22

10:12

$3.5(1-6)$

$3.3(1-6)$

$3.1(1-8)$

$3.4(1-8)$

5

2

9

7

$351(9-614)$

5

582 (38-2699)

14

617 (38-2699)

14

$8 / 1 / 10$

$5 / 4 / 13$

$4 / 2 / 12$

$12634(4028-$

32902)

\section{$23884(6350-$ 47894) \\ $17593(1586$ - 47649)}

25617 (8475 47894)

11271 (1208 46782)

\section{C1q:}

- Overall - $41 \%$ of $\mathrm{C} 1 \mathrm{q}$ positive failed vs $30 \%$ of $\mathrm{C} 1 \mathrm{q}$ negative $(p=0.53)$

- Specifically - $32 \%$ of C1q positive failed due to rejection vs $15 \%$ of $\mathrm{C} 1 \mathrm{q}$ negative $(p=0.28)$

- $64 \%$ of C1q positive suffered 1 or more episodes of AMR vs $25 \%$ of $C 1 q$ negative $(\mathrm{p}=0.015)$
Figure 1: C1q positive vs negative

Figure 2: C3d Positive vs Negative

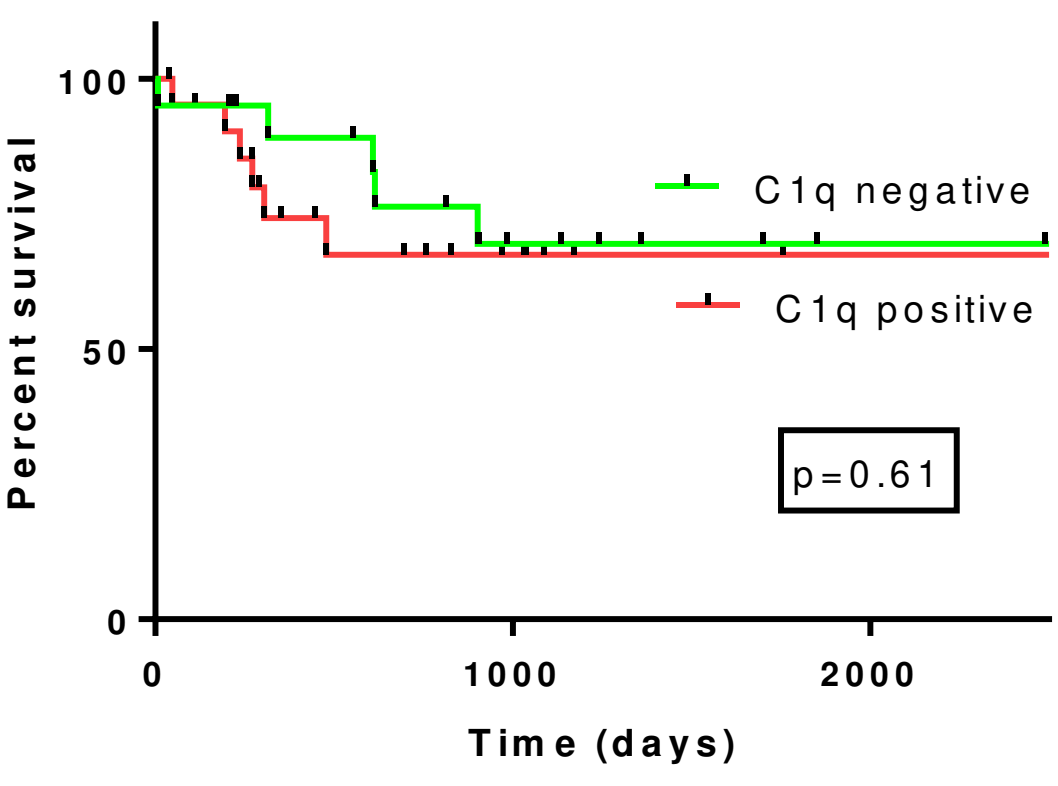

\section{C3d:}

- Overall - 44\% of C3d positive patients lost graft vs $25 \%$ C3d negative $(p=0.35)$

Specifically - 39\% of C3d positive lost graft to rejection vs $13 \%$ of C3d negative $(\mathrm{p}=0.07)$

- $78 \%$ of C3d positive suffered 1 or more episodes of AMR vs $21 \%$ of C3d negative $(p=0.0004)$

\section{Discussion and Conclusions:}

- Neither C1q nor C3d binding DSA pre transplant were significantly associated with poorer outcomes, however those patients with preformed DSA able to fix C3d showed a trend towards significant graft loss due to rejection $(p=0.07)$. Larger numbers are required to increase potential significance.

- The presence of pre-formed DSA capable of binding C1q or fixing C3d is significantly associated with post transplant episodes of AMR

- The use of complement detecting adaptations of single antigen beads can give useful information regarding the potential risk of graft loss and episodes of AMR post transplant.

- Of the two methods tested the C3d adaptation gave better trends of significance with both these outcomes.

- The addition of C3d testing to the pre-transplant repertoire could assist in donor selection, identification of higher risk antibody specificities and recipient risk assessment. 